\title{
Bericht
}

Simon Meier*

\section{Angemessenheit. Pragmatische Perspektiven auf ein linguistisches Bewertungskriterium}

\author{
Bericht zur Tagung der Arbeitsgemeinschaft \\ Linguistische Pragmatik (ALP e. V.) an der \\ Universität Leipzig am 3. März 2015
}

DOI 10.1515/zgl-2015-0017

In den jüngeren Bemühungen um eine linguistisch begründete Sprachkritik kommt dem Begriff der Angemessenheit eine zentrale Rolle zu. In Rückgriff auf die rhetorische Kategorie des aptum als gegenstands-, publikums- wie auch situationsbezogenes Regulativ wird Angemessenheit als dezidiert sprachgebrauchsbezogenes Bewertungskriterium gefasst, das insbesondere auf die Funktionalität sprachlicher Äußerungen und Texte abzielt (vgl. etwa Kienpointner 2005; Kilian/Niehr/Schiewe 2010: 39-45; Bücker/Diedrichsen/Spieß 2015: 13f.). Die Bewertung erfolgt dabei unter Einbezug etwa textsorten- oder medienspezifischer Faktoren sprachlicher Variation, für deren Erfassung nun gerade die pragmatisch orientierte Sprachwissenschaft geeignete Methoden und zahlreiche Anknüpfungspunkte bereitstellt. Insofern bot die Jahrestagung der Arbeitsgemeinschaft Linguistische Pragmatik einen passenden Rahmen, um sowohl die theoretischen wie methodischen Herausforderungen des Begriffs der Angemessenheit zu diskutieren als auch die vielfachen aktuellen Forschungen, die auch über den Bereich der linguistischen Sprachkritik hinaus mit dieser Kategorie operieren, zusammenzuführen. Denn gerade auch in didaktischen und angewandt-linguistischen Kontexten gewinnt der Begriff der Angemessenheit an Kontur (vgl. etwa Arendt/Kiesendahl 2011).

*Kontaktperson: Dr. Simon Meier: Institut für Sprache und Kommunikation, Fachgebiet Allgemeine Linguistik, Technische Universität Berlin, Straße des 17. Juni 135, D-10623 Berlin, E-Mail: simon.meier@tu-berlin.de 
Ein besonderer Mehrwert pragmatischer Zugriffe ist dabei auch in methodischer Hinsicht zu konstatieren, da etwa in interaktional orientierten Untersuchungen gezeigt werden kann, dass Angemessenheit nicht nur eine von außen eingebrachte Analysekategorie darstellt, sondern von den Teilnehmenden selbst als Maßstab für ihre eigenen Bewertungshandlungen herangezogen wird. Dies wiederum könnte als Grundlage für die Formulierung von kontextsensitiven Handlungsempfehlungen in mündlicher wie schriftlicher Kommunikation dienen. Die Vorträge der Tagung waren dementsprechend in vier thematische Blöcke eingeteilt: I) Theoretische Reflexionen, II) Aushandlungen von Angemessenheit, III) Angemessenheit und Gesprächskompetenz und IV) Formale Aspekte - Angemessenheit als Textqualität.

In ihrer Einführung umrissen die Organisatorinnen Birte Arendt, Jana Kiesendahl und Pavla Schäfer (alle Greifswald) die in der antiken Rhetorik liegenden Ursprünge wie auch die aktuelle pragmalinguistische Konturierung des Begriffs der Angemessenheit, vor allem aber die sich hieraus ergebenden Probleme. Die dabei angesprochenen Fragen etwa nach einer möglichen Operationalisierung dieser mehrdimensionalen Kategorie oder die Frage danach, wer nun nach welchen Maßstäben über Angemessenheit befindet oder befinden soll, welche Instanzen also als Normautoritäten gelten können, bildeten denn auch wiederkehrende Bezugspunkte in den Vorträgen der Tagung.

Den Anfang des ersten Blocks machte Thomas Niehr (Aachen), der in seinem theoretisch ausgerichteten Vortrag „Angemessenheit - eine Kategorie zwischen Präskriptivität und Inhaltsleere“ die offenkundige Unumstrittenheit von „Angemessenheit“ als sprachkritisches Schlagwort zum Anlass nahm, nach möglichen Präzisierungen $z u$ fragen, die gleichwohl nicht in simplifizierendes Rezeptwissen münden. Mit Blick auf die antike Fassung des aptum machte Niehr zunächst klar, dass Angemessenheit, anders als etwa grammatische Normen, nicht als starre Norm zu verstehen sei, aus der sich konkrete Handlungsanweisungen ableiten ließen. Deshalb sei Angemessenheit als meta-deontisches Konzept zu beschreiben, das zur Reflexion über Handlungsmöglichkeiten in bestimmten Situationen anweist. Somit ist Angemessenheit nur textsortenbezogen inhaltlich zu spezifizieren. Zugleich bedarf es aber auch eines (in der antiken Rhetorik besonders betonten) ethischen Korrektivs, um Angemessenheit nicht in bloßer Passung aufgehen zu lassen, die auch für manipulative Zwecke das Richtmaß abgeben könnte.

Auch Ulrike Krieg-Holz (Klagenfurt) und Lars Bülow (Passau) gingen in ihrem Vortrag „Angemessenheit als genuin pragmastilistisches Bewertungskriterium“ von der Annahme aus, dass Angemessenheit als eine Stilanforderung nur in Relation $\mathrm{zu}$ vorhandenen und historisch gewachsenen Textsortenmustern näher bestimmt werden kann. In kritischer Auseinandersetzung mit klassischen Ansätzen zur (normativen wie deskriptiven) Stilistik und mit kurzem Blick auf die 
moralische Komponente von Angemessenheit plädierten sie dafür, die Bewertungskategorie der Angemessenheit in der Frage nach der situativen Funktionalität von Texten zu fundieren. Sie stellten heraus, dass die Sprachspielkompetenz (vgl. Schneider 2009) sowie das Code-Switching eine wichtige Rolle für einen angemessenen Sprachgebrauch spielen. Letzteres machten sie auch am Beispiel des „Kiezdeutschen“ deutlich, dem sie sich über einen Rückblick auf die kritische Fachdiskussion über Wieses (2012) Forschungen näherten.

Die oben bereits erwähnte Frage nach den Entscheidungsinstanzen in Sachen Angemessenheit war Ausgangspunkt der drei folgenden, empirisch ausgerichteten Vorträge, die interaktionale Aushandlungen von Angemessenheit in verschiedenen kommunikativen Settings in den Blick nahmen, um so die Relevanz von Angemessenheit als normative Erwartung der TeilnehmerInnen selbst aufzeigen zu können. So zeigte Katharina König (Münster) in ihrem Vortrag „Die Verhandlung sprachlicher (Un-)Angemessenheit im Gespräch“ am Beispiel von Personenund Gruppenbezeichnungen, dass sich in Gesprächsphänomenen wie Verzögerungssignalen, Heckenausdrücken oder Reparaturverfahren ein Bewusstsein um die Unangemessenheit bestimmter Ausdrücke wie Zigeuner oder auch Menschen mit Migrationshintergrund manifestiert. Damit wird die Problematisierung und Stigmatisierung dieser Ausdrücke auch für den weiteren Gesprächsverlauf relevant gesetzt. Das Urteil der Unangemessenheit ist hier vornehmlich auf die Konnotationen von Ausdrücken bezogen, von denen sich die SprecherInnen mit subtilen Verfahren distanzieren, ohne auf die Ausdrücke gänzlich verzichten zu müssen.

Konstanze Marx (Berlin) analysierte in ihrem Vortrag „Zur Angemessenheit von Unangemessenheit - Die Banter-Funktion auf dem Online-Prüfstand“ ausführlich einen Mitschnitt einer Teamspeak-Konferenz, einer vornehmlich von Jugendlichen genutzten Form der computervermittelten spielbegleitenden Kommunikation. Der Mitschnitt gab eine Konferenz wieder, in die sich die Mutter eines der Teilnehmer einschaltete, um die offenbar mitgehörten Banteraktivitäten der Jugendlichen, also scherzhaft gerahmte Beleidigungen, zu kritisieren. Da Banter immer nur innerhalb einer bestimmten Gruppe akzeptiert und mithin angemessen ist, lassen sich, so die Überlegung, beim Hinzukommen einer nicht zugehörigen Teilnehmerin Aushandlungsprozesse von Angemessenheit, aber auch von Identitäten beobachten. Die Analyse zeigte, dass sich die Bedingungen der Onlinekommunikation wie räumliche Distanz und Anonymität der Teilnehmer deutlich in diese Aushandlungsprozesse einschreiben, indem es der Mutter nicht gelingt, eine kohärente Gesprächsidentität zu entwickeln. Ihre Kritikversuche bleiben somit hinter dem deutlich souveräneren Verhalten der Jugendlichen zurück.

Georg Albert und Nadine Hahn (Koblenz-Landau) nahmen in ihrem Vortrag „Erwartungen an die sprachliche Norm. Was in einem Online-Diskussionsforum 
als gute Ausdrucksweise ,durchgeht““ dagegen schriftliche Onlinekommunikation in den Blick. Anhand einer qualitativen Auswertung eines Korpus von Onlinediskussionen zeigten sie, wie hier in metasprachlichen Kommentaren Sprachkritik geübt und welche Aspekte und Bewertungskriterien dabei relevant gesetzt werden. Während Orthographie eine wichtige, auch mit Fragen der inhaltlichen Zustimmung bzw. Ablehnung interagierende Rolle spielt, werden morphosyntaktische Abweichungen kaum thematisiert. Auch Verstöße gegen Höflichkeitsnormen werden, oft mit Verweis auf die Netiquette, als unangemessen kritisiert. Gleichwohl stellt normgerechter Schriftsprachgebrauch keine Bedingung für erfolgreiche, d.h. anschlussfähige Diskussionsbeiträge dar. Aus der TeilnehmerInnenperspektive, so zeigen alle drei Vorträge dieses Blocks, scheint weniger Angemessenheit als Unangemessenheit die saliente Kategorie zu sein, die in der Interaktion metasprachlich thematisiert wird.

In den weiteren Vorträgen wurde auf verschiedene Weisen das Problem der Operationalisierung von Angemessenheit für forschungspraktische wie auch für didaktische Zwecke thematisiert. Den Block zum Verhältnis von Angemessenheit und Gesprächskompetenz eröffnete Kersten Sven Roth (Düsseldorf). In seinem Vortrag „Spielregeln des Sprechens - Was bedeutet ,Angemessenheit“ im Kompetenzbereich ,Sprechen und Zuhören“?“ unterzog er deutschdidaktische Texte wie Unterrichtsübungen, Lehrpläne und Bildungsstandards einer kritischen Betrachtung. Wiewohl die Fähigkeit zu angemessenem Gesprächsverhalten ein oft erwähntes Kompetenzziel darstellt, lässt sich hier ein praktisch nur schwer zu bewältigendes Geflecht konfligierender Angemessenheitsnormen ausmachen. Woran das zu erlernende Verhalten angemessen sein soll, bleibt oft unklar. Da es sich bei den im Unterricht geübten Gesprächsformen letztlich um klar geregelte Spiele mit autonomen Angemessenheitsordnungen handle, sei das Lernziel strenggenommen als Gesprächsspielregelkompetenz zu reformulieren.

Stefan Hauser (Zürich) und Martin Luginbühl (Neuchâtel) gaben in ihrem Vortrag „Aushandlung von Angemessenheit in argumentativen Gesprächen von Schulkindern“ einen Einblick in ein kürzlich angelaufenes Forschungsprojekt zum Erwerb von Argumentationskompetenz. Da sich Gesprächskompetenz überhaupt als Fähigkeit zu angemessenem Handeln in Gesprächen definieren lässt (vgl. Becker-Mrotzek/Brünner 2004: 29), können auch Argumentationen auf ihre Angemessenheit hin beurteilt werden. Dabei ist die Überlegung leitend, dass lokale Angemessenheitsstandards sich in der Gesprächssituation erst herausbilden, somit aber auch gesprächsanalytisch präzise rekonstruiert werden können. Anhand von Beispielanalysen (transkribierte Videoaufzeichnungen) zeigten die Referenten, dass schon Kinder über genaue, wenn auch aus Erwachsenensicht möglicherweise befremdlich wirkende Erwartungen und Vorstellungen verfügen, was für sie gute Gründe und mithin angemessene Argumentationen sind, 
und dies auch deutlich machen. Angemessenheit rückt auf diese Weise in die Nähe zum Konzept argumentativer Plausibilität.

Cordula Schwarze (Innsbruck) zeigte in ihrem Vortrag „Angemessenheit als Unterrichtsgegenstand und Bewertungsmaßstab in Auswertungsgesprächen in der Hochschule“ anhand von videographisch dokumentierten Gesprächen, wie sich aus den Angemessenheitsurteilen, die in hochschulischen Rhetorikkursen in der Bewertung von Vorträgen gefällt werden, didaktische Prinzipien ableiten lassen. Indem die Studierenden selbst Angemessenheit als relevante Kategorie einbringen, können Lehrende sie zum Unterrichtsgegenstand machen und die Gültigkeit von Normen als situationsbezogen und aushandelbar reflektieren. Der Vortrag machte somit auch die methodischen Herausforderungen bei der Operationalisierung von Angemessenheit deutlich. So wurden etwa die Länge und Häufigkeit von Pausen und das Sprechtempo als angemessenheitssensible Phänomene von den Studierenden diskutiert, ohne dass der Begriff der Angemessenheit explizit zur Bewertung herangezogen worden wäre. Hier stellte sich also die im Verlaufe der Tagung gleich mehrfach aufgegriffene Frage, an welchen sprachlichen Phänomenen Aushandlungen von Angemessenheit im einzelnen festgemacht werden kann, um die Kategorie nicht zu einer bloßen Sammelkategorie für Bewertungen jeglicher Art werden zu lassen.

Die Frage nach der Operationalisierbarkeit von Angemessenheit stellt sich in besonderem Maße für die Textproduktion, wie in den Vorträgen des abschließenden Blocks zur Angemessenheit als Textqualität dargelegt wurde. Bettina M. Bock (Leipzig) zeigte in ihrem Vortrag „Angemessenheit als Orientierungsmuster für sprachliches Handeln: Angemessene Texte für spezifische Zielgruppen schreiben“ anhand von Texten in „Leichter Sprache“, also speziell für Personen mit eingeschränkten kognitiven Fähigkeiten angepassten Texten, dass Verständlichkeit allein kein Maßstab für den gelungenen Transfer in Leichte Sprache sein kann. Die Kategorie der Angemessenheit biete demgegenüber den Vorteil, auch Kontextfaktoren wie textfunktionale und kommunikationsbereichsbezogene Aspekte miteinzubeziehen. Hieraus ließen sich schließlich auch sinnvollere Leitlinien für die Texterstellung als die derzeit üblichen, teils $\mathrm{zu}$ vagen, teils $\mathrm{zu}$ rigiden Regelkataloge ableiten. Zudem plädierte die Referentin dafür, neben der bloßen Funktionalität auch ästhetische Aspekte miteinzubeziehen, um der Künstlichkeit von Leichter Sprache entgegenzuwirken.

Abschließend brachte Sarah Brommer (Zürich) mit ihrem Vortrag „Sprachliche Muster als Indikator für die Angemessenheit eines Textes - Grundlagen einer automatisierten Stilanalyse“ den Vorschlag ein, korpuslinguistische Methoden für die objektive Bewertung der Qualität eines Textes nutzbar zu machen. Die Grundüberlegung war, dass sich die verbreitete Vorstellung davon, wie ein Text eines bestimmten Genres beschaffen sein sollte, im Wissen um typischen Sprach- 
gebrauch niederschlägt. Dieses textsortenbezogene Musterwissen lasse sich nun korpuslinguistisch anhand großer Textmengen errechnen. Der so gefundene Durchschnitt kann als Maßstab für die Beurteilung von Texten und der Stilkompetenz ihrer Verfasser dienen. Angemessenheit wird mithin als Stiladäquatheit enggeführt und die Bewertung von Texten findet in dieser objektiv nachweisbaren Textqualität ihre faktische Grundlage.

Die Tagung führte somit eindrucksvoll vor Augen, wie vielfältig die Fragestellungen sind, die rund um den Begriff der Angemessenheit bearbeitet werden, und wie etabliert und gesichert zugleich die grundlegenden Konturen dieses Konzepts sind, so dass ein derart kohärentes Tagungsprogramm möglich ist. Insgesamt zeigte sich, dass sich die Kategorie der Angemessenheit hervorragend dazu eignet, pragmalinguistisch orientierte Forschungen zur Sprachkritik, zur Stilistik wie auch zur Gesprächsanalyse und zur Textlinguistik zusammenzuführen. Dabei erwies sich insbesondere der systematische Einbezug der Situationsspezifik von Angemessenheitsstandards wie auch von Angemessenheitsurteilen als entscheidender Vorteil der pragmatischen Perspektive. Kontrovers wurde die Frage nach möglichen Operationalisierungen diskutiert, zumal in der Engführung von Angemessenheit nach Kriterien der Funktionalität die (in den antiken Entwürfen zentralen) moralischen Aspekte, aber auch die ästhetische Dimension sprachlicher Erzeugnisse oftmals aus dem Blick geraten. Als wünschenswert wurde in der Diskussion auch die historische Situierung von Angemessenheitskonzepten auch über den üblichen Referenzpunkt der antiken Rhetorik hinaus hervorgehoben. Auch das Verhältnis von Angemessenheitsurteilen zu anderen Formen der Bewertung (etwa der argumentativen Plausibilität, der ästhetischen Wohlgeformtheit usw.) bedarf noch weiterführender Diskussion.

Die Tagungsbeiträge werden in einem Themenheft der Zeitschrift Aptum. Zeitschrift für Sprachkritik und Sprachkultur (Heft 2/2015) publiziert, das im Herbst 2015 erscheinen soll.

Die nächste ALP-Tagung zum Thema „Sprachliche Verfestigungen und sprachlich Verfestigtes“ findet am 23.2.2016 in Konstanz statt. Informationen unter www.alp-verein.de

\section{Literatur}

Arendt, Birte/Kiesendahl, Jana (Hg.) (2011): Sprachkritik in der Schule. Theoretische Grundlagen und ihre praktische Relevanz, Göttingen: $\mathrm{V}+\mathrm{R}$ unipress.

Becker-Mrotzek, Michael/Brünner, Gisela (2004): „Der Erwerb kommunikativer Fähigkeiten. Kategorien und systematischer Überblick“, in: Michael Becker-Mrotzek/Gisela Brünner (Hg.): Analyse und Vermittlung von Gesprächskompetenz, Radolfzell: Verlag für Gesprächsforschung, 29-46. 
Bücker, Jörg/Diedrichsen, Elke/Spieß, Constanze (2015): „Perspektiven linguistischer Sprachkritik. Zur Einführung in den Sammelband“, in: Jörg Bücker/Elke Diedrichsen/Constanze Spieß (Hg.): Perspektiven linguistischer Sprachkritik, Stuttgart: ibidem, 7-22.

Kienpointner, Manfred (2005): „Dimensionen der Angemessenheit. Theoretische Fundierung und praktische Anwendung linguistischer Sprachkritik“, in: aptum. Zeitschrift für Sprachkritik und Sprachkultur 3, 193-219.

Kilian, Jörg/Niehr, Thomas/Schiewe, Jürgen (2010): Sprachkritik. Ansätze und Methoden der kritischen Sprachbetrachtung, Berlin, New York: de Gruyter.

Schneider, Jan Georg (2009): „Sprachkompetenz als Sprachspielkompetenz“, in: Mareike Buss/ Stephan Habscheid/Sabine Jautz/Frank Liedtke/Jan Georg Schneider (Hg.): Theatralität des sprachlichen Handelns. Eine Metaphorik zwischen Linguistik und Kulturwissenschaften München: Fink, 59-78.

Wiese, Heike (2012): Kiezdeutsch. Ein neuer Dialekt entsteht, München: Beck. 